\title{
ОБОБЩЕННОЕ ПРОГНОЗИРОВАНИЕ ПОКАЗАТЕЛЯ ПОГРУЗКИ ГРУЗОВ ПРИ ПЕРЕВОЗКЕ ЖЕЛЕЗНОДОРОЖНЫМ ТРАНСПОРТОМ
}

\author{
(С) 2020 Ю. М. Краковский ${ }^{凶}$, Н. Н. Попова
}

\author{
Иркутский государственный университет путей сообщения \\ ул. Черныпевского, 15, 664074 Иркутск, Российская Федерация
}

\begin{abstract}
Аннотация. Для повышения эффективности перевозочного процесса железнодорожным транспортом большое значение имеет прогнозирование показателей этого процесса, включая погрузку грузов. Учитывая, что перевозка грузов происходит в условиях неопределенности, задача прогнозирования является не тривиальной. В работе предлагается обобщенное прогнозирование показателя погрузки грузов при перевозке железнодорожным транспортом, когда используются три модели локальных показателей погрузки грузов с различными весами, а также учитываются сценарии развития перевозочного процесса. В качестве локальных показателей выбраны: а) значение, полученное по трехфакторной модели; б) значение, полученное по факторной модели, зависящей от времени; в) точечное экспертное суждение. Весовые коэффициенты получены с помощью метода анализа иерархий, использующего экспертные суждения в виде парных сравнений. Прогнозирование на основе совокупности моделей с учетом сценариев развития перевозочного процесса, является научной новизной работы. При апробации учитывались три сценария развития перевозочного процесса на Дальневосточной железной дороге. Использованы статистические данные с 2000 года по 2018 год, прогнозирование осуществлено на 2019 год. При первом сценарии учитывались только статистические данные, при втором дополнительно использовалась экспертная информация по оптимистическому варианту, при третьем использовалась экспертная информация по пессимистическому варианту. Знание фактической погрузки позволило подсчитать значения относительной погрешности обобщенного прогнозирования погрузки грузов по каждому сценарию. Можно отметить, что обобщенное прогнозирование показывает вполне хорошие результаты по всем трем сценариям, хотя сами модели прогнозирования дают существенную погрешность. Наиболее близкое значение получено при третьем сценарии.
\end{abstract}

Ключевые слова: регрессионные модели, экспертные суждения, сценарии развития, погрузка грузов.

\section{ВВЕДЕНИЕ}

Перевозки грузов железнодорожным транспортом во многих странах определяют развитие их экономики. Это относится и к России, т. к. исторически этот вид транспорта в грузовых перевозках у нее занимает ведущее место. В связи с этим, прогнозирование показателей перевозочного процесса железнодорожным транспортом имеет большое значение [1-3].

Краковский Юрий Мечеславович e-mail: 79149267772@yandex.ru
Для железнодорожной сети ОАО «РЖД» Дальневосточная железная дорога (ДВЖД) имеет особое значение, связанное с тем, что она является конечной и взаимодействует через морские порты и погранпереходы с другими странами.

В связи с этим, прогнозирование показателя погрузки грузов (млн.тонн) для этой дороги имеет существенное значение для повышения эффективности общего управления при перевозке грузов. Важность прогнозирования на основе регрессионных моделей отмечается в значительном числе работ, например [4-6].

c) (i) Контент доступен под лицензией Creative Commons Attribution 4.0 License.

The content is available under Creative Commons Attribution 4.0 License. 


\section{Ю. М. Краковский, Н. Н. Попова}

В условиях неопределенности перевозочного процесса это не тривиальная задача. В данной работе предложено и исследуется обобщенное прогнозирование погрузки грузов по статистическим данным ДВЖД с 2000 года по 2018 год, прогнозирование осуществлено на 2019 год.

При осуществлении обобщенного прогнозирования используются локальные модели прогнозирования: многофакторная модель, факторная модель погрузки грузов, зависящая от времени, и точечное экспертное значение. Эти значения затем преобразуются в обобщенное по линейной свертке с учетом значимости этих моделей. При определении весовых коэффициентов, характеризующих значимость моделей, используется метод анализа иерархий. Дополнительно обобщенное прогнозирование предложено осуществлять на основе сценарного подхода, который используется при прогнозировании и анализе социально-экономических систем [7]. Предложенное и апробированное обобщенное прогнозирование погрузки грузов с использованием сценарного подхода является новизной и практической значимостью работы.

\section{МНОГОФАКТОРНЫЕ И ТРЕНДОВЫЕ МОДЕЛИ ДЛЯ ПОГРУЗКИ ГРУЗОВ}

Перевозочный процесс железнодорожным транспортом оценивается достаточно большим числом факторов в той или иной мере связанных статистической связью с погрузкой $(l$, млн.т). Этими влияющими факторами являются [8]: статическая нагрузка, т/ ваг; средний вес грузового поезда, т; цена единицы перевезенного грузооборота, руб./млн. ткм; оборот вагона, сут.; простой местного вагона, час; участковая скорость, км/час; техническая скорость, км/час; производительность локомотива, тыс.ткм/сут; среднесуточный пробег локомотива, км/сут; грузооборот, млн.ткм.

Влияющие факторы существенно связаны между собой корреляционной связью. Используя коэффициенты парной корреляции [9], были оставлены только существенные из них, которых оказалось пять.
Создав пятифакторную модель и используя t-статистику, были оставлены значимые факторы, слабо связанные корреляционной связью. Это позволило получить адекватную трехфакторную модель первого порядка для погрузки грузов (ПГ), которая имеет вид

$$
l_{1}=51,972-1,680 \cdot t w-0,823 \cdot s+0,022 \cdot p, \text { (1) }
$$
где $t w-$ оборот вагона (ОбВаг); $s-$ участковая скорость (УчСкор); $p-$ производительность локомотива (ПрЛок). Значение критерия Фишера для модели (1) равно 19,3.

При использовании трехфакторной модели (1) сделано предположение, что все три фактора изменяются в требуемых практикой пределах.

Далее для ПГ создана адекватная факторная (трендовая) модель на основе статистической информации ДВЖД с 2000 года по 2018 год

$$
l_{2}=36,843 \cdot t^{0,106},
$$

где $t-$ время $(t=1,2,3, \ldots, 19)$. Значение критерия Фишера для модели (2) равно 21,3.

Аналогично созданы адекватные факторные модели для значимых факторов

$$
\begin{gathered}
t w=0,201 \cdot t+5,594, \\
s=0,012 \cdot t^{3}-0,409 \cdot t^{2}+30,635, \\
p=1206,100 \cdot t^{0,179} .
\end{gathered}
$$

Значение критерия Фишера для модели (3) равно 11,2 ; для модели (4) - 15,7 ; для модели (5) $-123,1$.

\section{СОЗДАНИЕ ТРЕНДОВЫХ МОДЕЛЕЙ С УЧЕТОМ ЭКСПЕРТНОЙ ИНФОРМАЦИИ}

Как правило, методы прогнозирования дают практические результаты, если тенденции изменения показателей сохраняются от предыдущих значений к будущим $[8,10]$. Это не всегда происходит, особенно для показателей, характеризующих перевозочный процесс железнодорожным транспортом, функционирующий в условиях неопределенности и риска [3]. В связи с этим, рекомендуется дополнительно к статистическим данным добавлять экспертные оценки.

В работе используются точечные экспертные оценки на прогнозируемый 2019 год по 
ПГ (l) и по значимым факторам $(t w, s, p)$. Эти оценки приведены в табл. 1 для двух вариантов (В): первого (1) и второго (2).

Первый вариант предполагает увеличение значений ПГ, ОбВаг, УчСкор и ПрЛок по сравнению с предыдущим 2018 годом (оптимистический вариант).

Второй - уменьшение значений ПГ, УчСкор и ПрЛок по сравнению с данными 2018 года (пессимистический вариант).

Отметим, что в 2018 году ПГ на ДВЖД равна 48,68 млн.т, ОбВаг равен 8,1 сут., УчСкор равна 36,6 км/час, ПрЛок 1994 тыс.ткм/сут. ОбВаг для второго варианта выбран немного выше, чем в 2018 году.

Используя статистические данные по ПГ и значимым факторам, а также экспертные оценки (табл. 1), дополнительно к моделям (2)-(5) в работе созданы новые факторные модели.

Факторные модели при первом варианте имеют вид

$$
\begin{gathered}
l_{2}=36,965 \cdot t^{0,104}, \\
t w=0,186 \cdot t+5,695, \\
s=0,011 \cdot t^{3}-0,403 \cdot t^{2}+3,853 \cdot t+30,697, \\
p=1209,800 \cdot t^{0,177} .
\end{gathered}
$$

Значение критерия Фишера для модели (6) равно 22,8 ; для модели (7) - 11,6; для модели (8) - 16,9; для модели (9) - 133,8.

Факторные модели при втором варианте имеют вид

$$
\begin{gathered}
l_{2}=37,040 \cdot t^{0,103}, \\
t w=0,181 \cdot t+5,735
\end{gathered}
$$

$$
\begin{gathered}
s=0,012 \cdot t^{3}-0,386 \cdot t^{2}+3,729 \cdot t+30,897, \\
p=1212,800 \cdot t^{0,175} .
\end{gathered}
$$

Значение критерия Фишера для модели (10) равно 21,9; для модели (11) - 10,7; для модели (12) - 17,0; для модели (13) - 126,4.

\section{ОБОБЩЕННОЕ РОГНОЗИРОВАНИЕ ПО- ГРУЗКИ С ПРИМЕНЕНИЕМ СЦЕНАРНОГО ПОДХОДА}

В табл. 2 приведены рекомендованные экспертами три сценария развития (Сц) перевозочного процесса для ДВЖД на 2019 год. Номер 2019 года относительно 2000 года равен $20(t=20)$.

В первом сценарии (1) используются только статистические данные (Ст). Поэтому при вычислении значения ПГ $l_{1}$ используется модель (1), значения факторов ( $t w, s, p)$ определяются по моделям (3), (4) и (5), соответственно. Значение ПГ $l_{2}$ вычисляется по модели (2). Для показателя $l_{3}$ экспертами принято значение 49,0 млн.т.

Во втором сценарии (2) используются статистические данные и экспертные оценки по варианту 1 (табл. 1) (Ст + В1). В этом сценарии при вычислении значения ПГ $l_{1}$ используется модель (1), но значения факторов $(t w, s, p)$ определяются по моделям (7), (8) и (9), соответственно. Значение погрузки $l_{2}$ вычисляется по модели (6). Для показателя $l_{3}$ экспертами принято значение 49,0 млн.т.

В третьем сценарии (3) используются статистические данные и экспертные оценки по

Таблица 1. Значения экспертных оценок

[Table 1. Values of expert assessments]

\begin{tabular}{|c|c|c|c|c|}
\hline B & $l$ & $t w$ & $s$ & $p$ \\
\hline 1 & 49,0 & 8,6 & 37,0 & 2000,0 \\
\hline 2 & 48,0 & 8,2 & 36,0 & 1950,0 \\
\hline
\end{tabular}

Таблица 2. Сиенарии развития перевозочного процесса

[Table 2. Development scenarios of the transportation process]

\begin{tabular}{|c|c|c|c|}
\hline Сц & $l_{1}$ & $l_{2}$ & $l_{3}$ \\
\hline 1 & Ст & Ст & 49,0 \\
\hline 2 & Ст + B1 & Ст + B1 & 49,0 \\
\hline 3 & Ст + B2 & С + В 2 & 48,0 \\
\hline
\end{tabular}


Таблииа 3. Матрица парных сравнений

[Table 3. Matrix of judgments]

\begin{tabular}{|c|c|c|c|}
\hline $\mathrm{M}$ & $l_{1}$ & $l_{2}$ & $l_{3}$ \\
\hline$l_{1}$ & 1 & $1 / 4$ & 3 \\
\hline$l_{2}$ & 4 & 1 & 5 \\
\hline$l_{3}$ & $1 / 3$ & $1 / 5$ & 1 \\
\hline
\end{tabular}

Таблица 4. Результаты апробации обобщенного прогнозирования погрузки

[Table 4. Testing results of generalized forecasting of cargo loading]

\begin{tabular}{|c|c|c|c|c|c|}
\hline Сц & $l_{1}(20)$ & $l_{2}(20)$ & $l_{3}(20)$ & $l_{c}(20)$ & $e, \%$ \\
\hline 1 & 47,542 & 50,613 & 49,000 & 49,756 & 3,44 \\
\hline 2 & 52,944 & 50,477 & 49,000 & 50,886 & 5,84 \\
\hline 3 & 42,582 & 50,492 & 48,000 & 48,410 & 0,69 \\
\hline
\end{tabular}

варианту 2 (табл. 1) (Ст + В2). В этом сценарии при вычислении значения ПГ $l_{1}$ используется модель (1), но значения факторов $(t w, s, p)$ определяются по моделям (11), (12) и (13), соответственно. Значение ПГ $l_{2}$ вычисляется по модели (10). Для показателя $l_{3}$ экспертами принято значение 48,0 млн.т.

Обобщенное прогнозирование предлагается проводить по линейной свертке вида

$$
l_{c}=\sum_{j=1}^{3} w_{j} \cdot l_{j},
$$

где $l_{1}-$ прогнозное на 2019 год значение ПГ по выбранному сценарию с использованием трехфакторной модели; $l_{2}-$ прогнозное на 2019 год значение ПГ по факторной модели; $l_{3}$ - экспертное прогнозное значение по ПГ на 2019 год по выбранному сценарию; $w_{j}$ весовые коэффициенты, учитывающие значимость перечисленных прогнозных моделей и значений ПГ.

В данном исследовании при вычислении весовых коэффициентов использован метод анализа иерархий [11]. Особенностью этого метода является создание экспертами матрицы суждений в виде парных сравнений. Эти суждения можно проверить на непротиворечивость, что является положительной стороной этого метода. В нашем случае объектами, которые сравниваются, являются модели и их значения по ПГ.

\section{АПРОБАЦИЯ ОБОБЩЕННОГО ПРОГНОЗИРОВАНИЯ ПОГРУЗКИ ГРУЗОВ}

Апробация обобщенного прогнозирования ПГ проведена с использованием экспертных оценок, приведенных в табл. 1, и сценариев развития перевозочного процесса, приведенных в табл. 2. Дополнительно была получена матрица парных сравнений (табл. 3), которую сформировали те же эксперты, которые подготовили экспертные оценки. Здесь M - перечисленные три модели вычисления значений ПГ.

Используя эту матрицу, по методу анализа иерархий получены следующие значения весовых коэффициентов

$$
w_{1}=0,226 ; w_{2}=0,673 ; w_{3}=0,101 .
$$

Отношение согласованности равно 0,073, оно, как и рекомендовано в этом методе, имеет значение меньше 0,1. Это позволяет отнестись с доверием к весовым коэффициентам (15).

В табл. 4 приведены результаты расчетов по рекомендованным сценариям. Напомним, что 20 - это номер 2019 года. В правой колонке приведены значения относительной погрешности обобщенного прогнозирования ПГ относительно фактического значения в процентах (e).

Приведем результаты расчетов по первому сценарию:

по формуле (3) прогнозное значение на 2019 год для ОбВаг равно 9,6 сут.; 
по формуле (4) прогнозное значение на 2019 год для УчСкор равно 40,9 км/час.;

по формуле (5) прогнозное значение на 2019 год для ПрЛок равно 2061,9 тыс.ткм/сут.

Используя эти значения, по формуле (1) определяем прогнозное значение ПГ $\left(l_{1}\right)$, которое приведено в табл. 4 (47,542 млн.т). Используя формулу (2), определяем прогнозное значение ПГ $\left(l_{2}\right)$, которое приведено в табл. 4 (50,613 млн.т).

Эксперты по первому сценарию спрогнозировали значение ПГ $\left(l_{3}\right)$, приведенное в таблице 4 (49,0 млн.т). По формуле (14) с учетом (15) было получено обобщенное прогнозное значение по ПГ $\left(l_{c}\right)$, которое приведено в таблице 4 (49,756 млн.т).

Аналогично были проведены расчеты по второму и третьему сценариям.

Подчеркнем, что данные расчеты проведены во второй половине 2019 года, когда реальных данных по ПГ на ДВЖД за 2019 еще не было.

В настоящий момент известно, что:

ПГ в 2019 году на ДВЖД равна 48,076 млн.т, она немного уменьшилась по сравнению с 2018 годом;

ОбВаг равен 8,8 сут., он вырос по сравнению с 2018 годом;

УчСкор равна 37,6 км/час, она увеличилась по сравнению с 2018 годом;

ПрЛок равна 1968 тыс.ткм/сут, она уменьшилась по сравнению с 2018 годом.

Таким образом, на фактических значениях подтверждается неопределенность перевозочного процесса железнодорожным транспортам.

Знание фактической погрузки позволило подсчитать значения относительной погрешности обобщенного прогнозирования ПГ по каждому сценарию (табл. 4).

При этом необходимо отметить, что обобщенное прогнозирование, при котором используются значения ПГ по трехфакторной и по факторной моделям, а также экспертное значение, показывает вполне хорошие результаты по всем трем сценариям.

Наиболее близкое значение получено при третьем сценарии, когда, помимо статистиче- ской информации, используются экспертные оценки на 2019 год по второму варианту.

\section{ЗАКЛЮЧЕНИЕ}

Разработана и апробирована технология обобщенного прогнозирования ПГ с применением различных сценариев, использующих статистическую и экспертную информацию. Обобщенное прогнозирование основано на трех значениях ПГ с различными весами: а) значение, полученное по трехфакторной модели; б) значение, полученное по факторной модели, зависящей от времени; в) точечное экспертное суждение. Весовые коэффициенты получены с помощью метода анализа иерархий, использующего экспертные суждения.

Использование обобщенного прогнозирования показателя погрузки грузов является научной новизной работы.

При апробации использовались три сценария развития перевозочного процесса на ДВЖД. Для этого использованы статистические данные с 2000 года по 2018 год, прогнозирование осуществлено на 2019 год. Показана хорошая практическая точность этого прогнозирования по всем трем сценариям, хотя сами модели прогнозирования дают существенную погрешность. Наиболее близкое значение получено при третьем сценарии, когда, помимо статистической информации, используются экспертные оценки на 2019 год по второму варианту.

\section{КОНФЛИКТ ИНТЕРЕСОВ}

Авторы декларируют отсутствие явных и потенциальных конфликтов интересов, связанных с публикацией настоящей статьи.

\section{СПИСОК ЛИТЕРАТУРЫ}

1. Начигин, B. А. Процедура выбора предпочтительного сценария развития перевозочного процесса / В. А. Начигин, В. Ф. Фролов // Известия Транссиба. - 2014. - № 1. - С. 109115.

2. Краковский, Ю. М. Прогнозирование показателей грузовых перевозок Улан-Батор- 


\section{Ю. М. Краковский, Н. Н. Попова}

ской железной дороги / Ю. М. Краковский, И. А. Домбровский // Современные технологии. Системный анализ. Моделирование. 2013. - № 4 (40). - С. 225-228.

3. Краковский, Ю. М. Моделирование перевозочного процесса железнодорожным транспортом: анализ, прогнозирование, риски / Ю. М. Краковский, С. К. Каргапольцев, В. А. Начигин; под ред. проф. Ю. М. Краковского. - Санкт-Петербург: «ЛИТЕО», 2018. $240 \mathrm{c}$.

4. Базилевский, М. П. Многокритериальный подход к построению двухфакторных полносвязных регрессий на примере моделирования ВВП России / М. П. Базилевский // Вестник ВГУ, серия: системный анализ и информационные технологии. - 2020. - № 1. C. 99-109.

5. Базилевский, М. П. Исследование двухфакторной модели полносвязной линейной регрессии / М. П. Базилевский // Моделирование, оптимизация и информационные технологии. - 2019. - Т. 7, № 2 (25). - С. 80-96.

6. Кирилюк, И. Л. Модели производственных функций для российской экономики /
И. Л. Кирилюк // Компьютерные исследования и моделирование. - 2013. - Т. 5, № 2. C. $293-312$.

7. Шульи, В. Л. Модели и методы анализа и синтеза сценариев развития социально-экономических систем / В. Л. Шульц, В. В. Кульба. - М. : Наука, 2012. - Кн. 1. - 358 с.

8. Краковский, Ю. М. Прогнозирование базовых показателей перевозочного процесса на основе сценарного подхода / Ю. М. Краковский, А. Н. Лузгин // Прикладная информатика. - 2017. - Т. 12, № 2(68). - С. 29-36.

9. Носко, В. П. Эконометрика / В. П. Носко. - М. : Издательский дом «Дело» РАНХиГС, 2011. - Кн. 1. - 672 с.

10. Давааням, Тамир. Комплексное прогнозирование базовых показателей перевозочного процесса / Тамир Давааням, Ю. М. Краковский // Современные технологии. Системный анализ. Моделирование. 2016. - №3(51). - С. 179-184.

11. Саати, Т. Принятие решений. Метод анализа иерархий / Т. Саати. - М.: Радио и связь, 1993. - 320 с.

Краковский Юрий Мечеславович - д-р техн. наук, проф., профессор кафедры «Информационные системы и защита информации», Иркутский государственный университет путей сообщения.

E-mail: 79149267772@yandex.ru

ORCID iD:https://orcid.org/0000-0003-4876-0618

Попова Наталья Николаевна - аспирант кафедры «Информационные системы и защита информации», Иркутский государственный университет путей сообщения.

E-mail: dayseven.93@yandex.ru

ORCID iD:https://orcid.org/ 0000-0002-3751-2638 


\title{
GENERALIZED FORECASTING OF CARGO INDICATORS FOR RAIL TRANSPORTATION
}

\author{
(C) 2020 Y. M. Krakovsky ${ }^{\bowtie}$, N. N. Popova \\ Irkutsk State Transport University \\ 15, Chernyshevsky Street, 664074 Irkutsk, Russian Federation
}

\begin{abstract}
Annotation. To improve the efficiency of the rail transportation process it is important to forecast its indicators, including cargo loading. Given that the transportation of goods occurs under conditions of uncertainty, the task of forecasting is not trivial. This paper proposes a generalised forecasting method for the cargo indicators of rail transportation, which uses three models of local indicators of cargo with different weights and takes into account the scenarios of the transportation process. The following indicators were selected as local indicators: a) the value obtained by the three-factor model; b) the value obtained by the time-dependent factor model; c) selective expert judgement. The weight coefficients were determined by the analytic hierarchy process which uses expert judgement in the form of pairwise comparisons. This approach is a scientific novelty. Testing involved three scenarios of the transportation process on the Far Eastern Railway. Statistical data from 2000 to 2018 were used, and forecasting for 2019 was made. The first scenario only considered statistical data, the second scenario also used expert information based on the optimistic variant, and the third scenario used expert information based on the pessimistic variant. Knowledge of actual cargo loading allowed us to calculate the relative error values of the generalised forecasted cargo loading for each scenario. It can be noted that the method of generalised forecasting shows quite good results for all the three scenarios, although the forecasting models themselves give a significant error. The closest values were obtained with the third scenario.
\end{abstract}

Keywords: regression models, expert judgement, scenarios, cargo loading.

\section{CONFLICT OF INTEREST}

The authors declare the absence of obvious and potential conflicts of interest related to the pub-lication of this article.

\section{REFERENCES}

1. Nachigin V. A., Frolov V. F. Preferred selection procedure scenario development transportation process. Journal of Transsib Railway Studies.2014. No 1. pp. 109-115.

2. Krakovsky Y. M., Dombrovsky I. A. Forecasting indicators of Ulaanbaatar railway freight traffic. Modern technologies. System analysis. Modeling. 2013. No. 4 (40). pp. 225-228.

Krakovsky Yuri M.

e-mail: 79149267772@yandex.ru
3. Krakovsky Y. M., Kargapoltsev S. K., Nachigin V. A. Modeling of railway the trans-portation process by rail: analysis, forecasting, risks. Ed. by Y.M. Krakovsky. St. Petersburg : LITEO, 2018. $240 \mathrm{p}$.

4. Basilevskiy M. P. Multi-criteria ap-proach to the construction of fully connected two-factor regressions based on the modeling of the GDP of Russia. Scientific journal Proceed-ings of VSU, Series: system analysis and infor-mation technology. 2020. No. 1. pp. 99-109.

5. Basilevskiy M. P. Research of a two-factor fully connected linear regression model. Modeling, optimization and information tech-nology. 2019. V. 25, No. 2. pp. 80-96.

6. Kirilyuk I. L. Models of production functions for the Russian economy. Computer Research and Modeling. 2013. V. 5, No. 2. pp. 293312. 


\section{Ю. М. Краковский, Н. Н. Попова}

7. Shultz V. L., Kul'ba V. V. Models and methods of analysis and synthesis of scenarios for the development of socio-economic systems. Moscow: Nauka, 2012. Book. 1. 358 p.

8. Krakovsky Y. M., Luzgin A. N. Forecast-ing of basic indicators of the transportation pro-cess based on a scenario approach. Journal of Applied Informatics. 2017. V. 12. No. 2(68). pp. 29-36.

9. Nosko V. P. Econometrics. Moscow: Izdatel'skij dom «Delo» RAN-HiGS, 2011. Book.1. $672 \mathrm{p}$.

Krakovsky Yuri M. - DSc in Technical Sciences, Professor, Department of Information Systems and Information Security, Irkutsk State Transport University.

E-mail: 79149267772@yandex.ru

ORCID iD:https://orcid.org/0000-0003-4876-0618

Popova Natalya N. - PhD student, the Department of Information Systems and Information Security, Irkutsk State Transport University.

E-mail: dayseven.93@yandex.ru

ORCID iD:https://orcid.org/ 0000-0002-3751-2638
10. Davaanyam T., Krakovsky Y. M. Inte-grated forecasting of the main of indicators of transportation process. Modern technologies. System analysis. Modeling. 2016. No. 3(51). pp. 179-184.

11. Saati T. Decision-making. Analytic hierarchy process. Moscow : Radio i svyaz, 1993. 320 p. 